\title{
Vertex Cover Approximations on Random Graphs
}

\author{
Eyjolfur Asgeirsson ${ }^{1}$ and Cliff Stein ${ }^{2}$ \\ ${ }^{1}$ Reykjavik University, Reykjavik, Iceland \\ eyjo@ru.is \\ ${ }^{2}$ Department of IEOR, Columbia University, New York, NY \\ cliff@ieor.columbia.edu
}

\begin{abstract}
The vertex cover problem is a classical NP-complete problem for which the best worst-case approximation ratio is $2-o(1)$. In this paper, we use a collection of simple graph transformations, each of which guarantees an approximation ratio of $\frac{3}{2}$, to find approximate vertex covers for a large collection of randomly generated graphs. These reductions are extremely fast and even though they, by themselves are not guaranteed to find a vertex cover, we manage to find a $\frac{3}{2}$-approximate vertex cover for almost every single random graph we generate.
\end{abstract}

\section{Introduction}

In 1972, a year after Cook 2 formalized the notion of NP-completeness and proved that the boolean satisfiability problem is NP-complete, Karp [11] showed that 21 diverse problems from graph theory and combinatorics are NP-complete. One problem from this set of $21 \mathrm{NP}$-complete problems is the vertex cover problem. A vertex cover of a graph $G=(V, E)$ is a subset of the vertices, $C \subseteq V$, such that each edge $e \in E$ has at least one endpoint in $C$. The objective is to minimize the size of the vertex cover.

The best known approximation ratio for vertex cover is $2-\Theta\left(\frac{1}{\sqrt{\log n}}\right)$. This approximation ratio is achieved by an algorithm by Karakostas 10 . It is NP-hard to approximate minimum vertex cover within any factor smaller than 1.36 [5]. A simple greedy algorithm gives a 2-approximation for the vertex cover problem [6] and several people conjecture that there does not exist an algorithm with a fixed approximation ratio better than $2[9]$. Other work on vertex cover includes 4[12]8.

In 2005, Asgeirsson and Stein [1] introduced the idea of finding an approximate vertex cover using simple graph reductions. These reductions are not guaranteed to find a vertex cover, but if successful give a $\frac{3}{2}$-approximate vertex cover (and usually one that is significantly better). Asgeirsson and Stein showed that by using the heuristic, they could find a $\frac{3}{2}$-approximate vertex cover for every single graph that they tried, which included every graph that they found in the literature on vertex cover.

In this paper, we will extend and improve the results of Asgeirsson and Stein in several ways. First, we will introduce a new graph reduction, which we call 
the almost bipartite reduction. We will then analyze it, and relate it to the other graph reductions introduced in [1]. In particular, we will show that there are classes of graphs for which the heuristics of [1 do not find a vertex cover, but the addition of the almost bipartite reduction allows us to find a vertex cover. Second, we will extend the class of graphs analyzed to include random graphs. The random graphs that we use are based on the simple random graph model by Erdös and Rényi [7], where we start with a fixed set of vertices and add edges to the graph based on a edge probability parameter. Finally, we give a more detailed analysis than [1, and analyze the performance of each graph reduction with respect to various factors, such as the density of the graph and the number of vertices. We will also show that the choice of reductions depends on the edge probabilities, and that for certain probabilities, the almost-bipartite reductions is very useful.

The graph reductions do not guarantee that we will find an approximate vertex cover, but in our experiments we managed to find a $\frac{3}{2}$-approximate vertex cover for almost every single graph we tried. For the graphs where we know the size of the minimum vertex cover, the actual approximation ratio was usually much lower than $\frac{3}{2}$; in many cases the vertex cover we found was either optimal or very close to optimal.

\section{Graph Reductions}

Asgeirsson and Stein [1] introduced a heuristic, based on simple graph reductions, that tries to find a $\frac{3}{2}$-approximate vertex cover by breaking the graph into smaller and easier subproblems. The graph reductions focus on special graph structures and their goal is to remove a set $V^{\prime}$ of vertices and a set $E^{\prime}$ of edges from the graph by finding a vertex cover for the subgraph induced by the set $V^{\prime}$. The reductions are designed so that when we combine the vertex covers for all the subgraphs, we get an approximate vertex cover for the original graph. The approximation ratio of the vertex cover is equal to maximum approximation ratio over all the reductions that are used. Unless otherwise stated, we will only use reductions with approximation ratio no more than $\frac{3}{2}$, so the resulting vertex cover for the original graph, if we succeed in finding one, will have an approximation ratio of $\frac{3}{2}$. Before we list the graph reductions, we need the following definitions [1]:

Definition 1. An optimal graph reduction is a mapping from a graph $G=$ $(V, E)$ to a graph $G^{\prime}=\left(V^{\prime}, E^{\prime}\right)$ with the property that if we have an optimal vertex cover $V C_{\text {opt }}^{\prime}$ for $G^{\prime}$ then we can create an optimal vertex cover for the original graph $G$ from $V C_{\text {opt }}^{\prime}$ and from the graph reductions that we performed on the graphs.

Definition 2. A $\rho$-approximating graph reduction is a mapping from a graph $G=(V, E)$ to a graph $G^{\prime}=\left(V^{\prime}, E^{\prime}\right)$ such that if we have an optimal vertex cover $V C$ for $G^{\prime}$ then we can create a $\rho$-approximate vertex cover for $G$ from $V C$ and from the graph reductions that we performed on the graph. 
We will use both optimal graph reductions and approximate graph reductions. Most of the approximate reductions have approximation ratio $\rho \leq \frac{3}{2}$, but we will introduce reductions that have higher approximation ratios. An operation we will use extensively is a vertex contraction.

Definition 3. The contraction of a set of vertices $v_{1}, \ldots, v_{k}$ to a new vertex $v$ is an operation where we replace the vertices $v_{1}, \ldots, v_{k}$ with a new vertex $v$, delete all edges between removed vertices and replace each edge $\left(v_{i}, u\right)$ with an edge $(v, u)$. The set of vertices adjacent to $v$ is the union of the vertices that were adjacent to $v_{1}, \ldots, v_{k}$. If we use the mapping to $v$ to find an approximate vertex cover for the original graph we call $v$ an approximated vertex.

When we perform a vertex contraction, we replace multiple edges that might appear with a single edge and encode information about the contracted vertices and adjacent edges so that we can recreate them later to get the original graph.

Most of the graph reductions that we use are defined in [1]. For completeness, we list the graph reductions here along with a brief overview. The name we use for each reduction refers to the graph structure that the reduction uses to attack the graph. Since we are interested in finding an approximate vertex cover, and each reduction we use has a particular approximation ratio, we group the reductions into optimal and approximate graph reductions, according to Definitions 1 and2.

\section{Optimal Graph Reductions}

- Degree-0 vertices: Removes a single vertex of degree-0 and no edges.

- Degree-1 vertices: There is an optimal cover that includes the neighbor and not the degree-1 vertex. This reduction removes the degree-1 vertex and its neighbor along with the edge between them. Edges connected to the neighbor are also removed.

- Degree-2 vertices with adjacent neighbors: There is an optimal vertex cover that includes both neighbors and not the degree- 2 vertex. We remove the degree- 2 vertex and both its neighbors along with all edges adjacent to these three vertices.

- Degree-2 vertices with non-adjacent neighbors: Contracts the degree2 vertex and its neighbors into a single vertex for a new graph $G^{\prime}$. Removes at least two edges. The contracted vertex is not approximated. If the optimal cover for the new graph includes the contracted vertex, then the optimal cover for the original graph includes both neighbors and not the degree-2 vertex. If the optimal cover for $G^{\prime}$ does not include the contracted vertex then the optimal vertex cover for the original graph includes the degree-2 vertex and not the neighbors.

- Extended Network Flow: Removes all vertices corresponding to non-half variables in the optimal solution to the LP-relaxation of the vertex cover problem. We also try to fix each variable equal to 1 and resolve in order to get an optimal LP solution with more non-half variables. If the optimal solution with a variable fixed as 1 is equal to the optimal solution with no fixed variables, we get a new optimal solution with more non-half variables that we can remove. 


\section{Approximate Graph Reductions}

- Triangle elimination: Removes the three vertices in the triangle and all edges connected to these vertices. Any cover must use at least 2 of the vertices, so by using all 3 we get a $\frac{3}{2}$-approximation.

- Degree-3 vertices: Contracts the degree-3 vertex and its neighbor into a single approximated vertex. Removes at least three edges. This is a $\frac{3}{2}-$ approximation.

- Four-cycles: Contracts the four vertices from a chordless cycle of length four into two approximated vertices. Removes at least three edges. The fourcycle reduction is a $\frac{4}{3}$-approximate graph reduction.

- Five-cycles: Removes the five vertices from a cycle of length five and all edges connected to these vertices. The five-cycle reduction is a $\frac{5}{3}$-approximate reduction. We will only use this reduction if we cannot find a $\frac{3}{2}$-approximate vertex cover by using the other reductions.

- Six-cycles: Contracts the six vertices in a chordless cycle of length six into two approximated vertices and removes at least five edges. The approximation ratio of the 6 -cycle reduction is $\frac{3}{2}$.

\subsection{New Graph Reduction: Almost-Bipartite}

When we are trying to solve NP-hard problems it is often helpful to look for special cases. For many problems, while the general problem is NP-hard, there are special cases that yield an optimal solution in polynomial time. One such example is the vertex cover for bipartite graphs. The vertex cover problem is well known to be NP-hard for general graphs but for the special case of bipartite graphs, we can find an optimal vertex cover in polynomial time using maximum matching. It is also well known that the size of the minimum vertex cover for bipartite graphs is equal to the size of the maximum matching [3].

We can use the fact that it is easy to find an optimal vertex cover for a bipartite graph to try to find approximate vertex covers for general graphs. If we have a lower bound of $k$ for the optimal vertex cover, we know that any vertex cover of size no more than $p k$ is a $p$-approximate vertex cover.

The almost bipartite method is as follows: We partition the vertices of the graph into three sets, $A, B$ and $C$, such that the subgraph induced by the first two sets of vertices, $A$ and $B$, will be bipartite. The last set, $C$, will include all vertices that violate the bipartite property if they are added to the subgraph, i.e. each vertex in the set $C$ has neighbors in both sets $A$ and $B$.

Claim. Let $G=(V, E)$ be a graph. Assume we partition $V$ into three sets, $A$, $B$ and $C$, with the property that the subgraph induced by the sets $A$ and $B$ is bipartite. Let $G_{A B}$ be the bipartite subgraph induced by the sets $A$ and $B$ and let $\mathrm{VC}_{G_{A B}}^{*}$ be an optimal vertex cover for $G_{A B}$. Also let $\mathrm{VC}_{L B}$ be a lower bound on the size of the optimal vertex cover for the original graph, and let $p$ be the approximation ratio that we want to achieve. Then, if $\left|\mathrm{VC}_{G_{A B}}^{*}\right|+|C| \leq p \mathrm{VC}_{L B}$, the set $\mathrm{VC}_{G_{A B}}^{*} \cup C$ is a $p$-approximate vertex cover for $G$. 
Proof. The vertex cover $\mathrm{VC}_{G_{A B}}^{*}$ covers all edges in $G_{A B}$ and all other edges in $G$ are covered by the set $C$, so $\mathrm{VC}_{G_{A B}}^{*} \cup C$ is a feasible vertex cover for $G$. If $\mathrm{VC}_{L B}$ is an lower bound on the optimal vertex cover for $G$, then any feasible vertex cover whose size is smaller than $p \mathrm{VC}_{L B}$ is a $p$-approximate vertex cover for $G$. We know that $\mathrm{VC}_{L B} \leq\left|\mathrm{VC}^{*}\right|$, where $\mathrm{VC}^{*}$ is an optimal vertex cover for $G$. If the sum of the size of the set $C$, and the size of the optimal vertex cover on the bipartite subgraph $G_{A B}$ is smaller than $p \mathrm{VC}_{L B}$, then $\left|\mathrm{VC}_{G_{A B}}^{*}\right|+|C| \leq p \mathrm{VC}_{L B} \leq p\left|\mathrm{VC}^{*}\right|$ and $\mathrm{VC}_{G_{A B}}^{*} \cup C$ is a $p$-approximate vertex cover for $G$.

In our experiments, the approximation ratio $p$ is usually equal to $3 / 2$. The almost-bipartite method succeeds if the size of the optimal vertex cover on the subgraph induced by the sets $A$ and $B$ plus the size of set $C$ is less than the upper limit on the size of the approximate vertex cover, i.e. if $\left|\mathrm{VC}_{G_{A B}}^{*}\right|+|C| \leq p \mathrm{VC}_{L B}$.

The problem of finding a maximum sized bipartite subgraph in a graph is NP-hard [4. Finding a maximum sized bipartite subgraph is a special case of the problem of finding a maximum induced subgraph with property $P$, where property $P$ is hereditary and non-trivial. A property $P$ of graph $G$ is hereditary if every subgraph of $G$ also satisfies $P$. In our case, the property $P$ is the bipartite property. The problem of finding a maximum induced subgraph with property $P$ is approximable within $O\left(\frac{n}{\log (n)}\right)$ where $n$ is the number of vertices. However, the problem is not approximable within $n^{\epsilon}$ for some $\epsilon>0$ unless $P=N P$.

Therefore, we settled for heuristics to find the almost-bipartite subgraph. We tested a few different implementations of the almost-bipartite method. However, the comparison between these methods indicated that there was not a large difference between the various partitioning methods. We settled on a greedy method in which we start by adding a single vertex to set $A$ and then focus on each set in turn, switching between sets only when we have processed every vertex in the current set of vertices. A vertex is processed by iterating through all its neighbors and placing them in the appropriate sets. The unprocessed vertices in each set are selected in the order in which they are added to the set. If there are unprocessed vertices after we process all vertices in the sets $A$ and $B$, we add a randomly selected unprocessed vertex to set $A$ and continue as before. If the algorithm fails, we try starting from another vertex until we either find an approximate vertex cover or until we have tried starting from all the vertices, in which case the algorithm fails.

It is somewhat tricky to combine the almost bipartite method with the other reductions since the almost bipartite method cannot work on graphs that contain approximated vertices. The almost bipartite method also needs a good lower bound on the size of the optimal vertex cover in order to work. However, if we can overcome these obstacles then the almost-bipartite reduction is very powerful because when it succeeds, it completely solves the graph.

\subsection{The Automated Order of Reductions}

The automated order of the graph reductions is the same order that was suggested in [1, with the addition of the almost bipartite method. We run the 


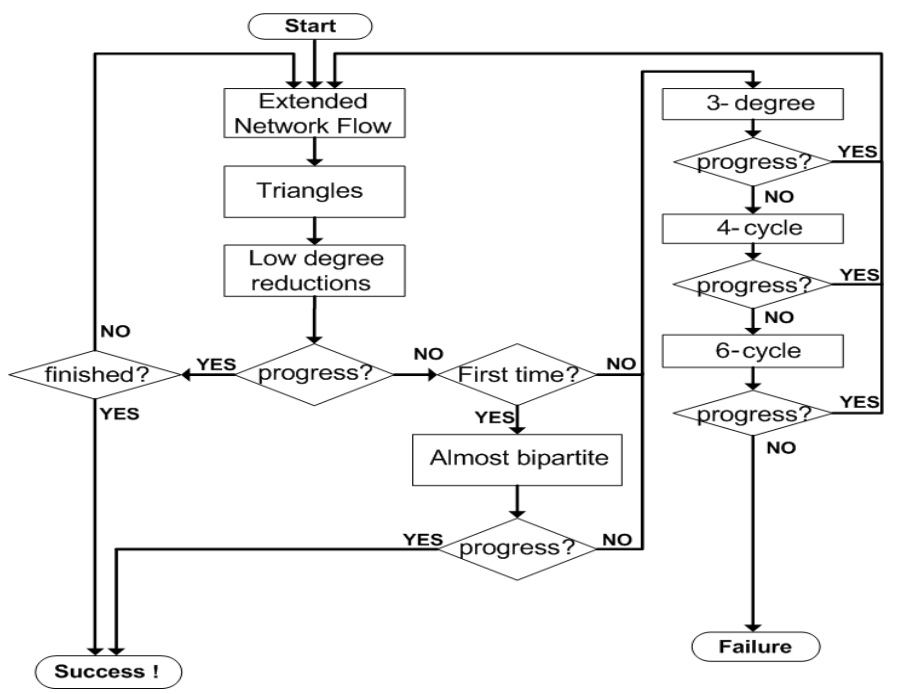

Fig. 1. A flowchart showing the automated order of the reductions

extended network flow method, triangle elimination and low-degree in a loop until we find a solution or no improvements are made during an iteration. If the graph is not empty and we have not created any approximated vertices, we try the almost bipartite reduction. If the almost bipartite method does not solve the graph, we try the 3-degree, 4-cycle and 6-cycle reductions, stopping as soon as any one of them makes some progress and return to the original loop. The stopping criteria is either having processed all the vertices from the graph which gives us a vertex cover, or running 3-degree, 4-cycle and 6-cycle without any improvements. In that case we stop and must use some other methods, such as branch-and-bound, to get a solution. If we find a solution then the final step in the algorithm is to use a simple greedy algorithm to eliminate unnecessary vertices from the cover. In our experiments we almost never had to resort to branch-and-bound, our algorithm managed to solve almost every single graph we found. The flowchart for the automated order is shown in Figure 1] The 5cycle reduction is not included in Figure 1 since its approximation ratio is higher and we only use it if we cannot find a $\frac{3}{2}$-approximate vertex cover by using the other reductions.

\section{Experiments on Random Graphs}

The random graphs we generated are based on the simple random graph model by Erdös and Rényi [7]. We start with fixed set of vertices $V$ where $|V|=n$. First we randomly select an edge density parameter $\rho$ such that $0<\rho<1$. Then for each possible edge $e$, we add the edge with probability $\rho$, i.e. for any 
possible edge, we generate a random number between 0 and 1 . If this randomly generated number is less than $\rho$, we add the edge to the graph, while if the randomly generated number is greater than $\rho$ the edge is not included in the graph. We try this once for every possible edge, i.e. for any pair of vertices $v_{i}, v_{j} \in V$.

We used four different sets of vertices, with 200, 500, 1000 and 10,000 vertices. We generated 20,000,000 random graphs on 200 vertices, 1,000,000 random graphs on 500 vertices and 100,000 random graphs on 1000 vertices. Finally we generated 2000 sparse random graphs on 10,000 vertices. By using our reductions, we managed to find $\frac{3}{2}$-approximate vertex cover for every randomly generated graph with 1000 vertices or less. Because we solved every graph with at most 1000 vertices, we will focus on the performance of each reduction and show how effective each reduction is for the smaller random graphs, based on the edge probability. The large graphs, with 10,000 vertices, are a special case since the graphs are all sparse and we only have a relatively small collection. We will analyze the large graphs separately.

\subsection{Using the Almost Bipartite Reduction}

The almost bipartite method differs from our other reductions because it is guaranteed to finish the graph if it works, and the graph needs to fulfill specific conditions in order for us to use it. We therefore wanted to understand how the almost bipartite method relates to the other reductions and how important it is when we are trying to find approximate vertex covers. The majority of the graphs that we generated were solved by using only the triangle elimination, the extended network flow method and the low degree reductions, but there were some graphs that could not be solved without using the almost bipartite method. Figure 2 shows the fraction of graphs that were solved as a function of
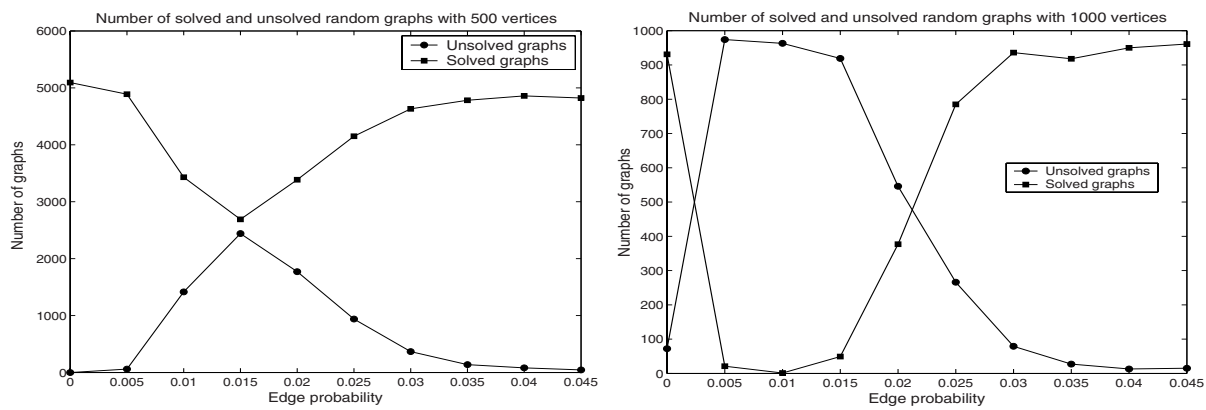

Fig. 2. The number of random graphs that were solved without using the almost bipartite method and the number of graphs that could not be solved without the almost bipartite method. The first graph shows the results for randomly generated graphs on 500 vertices and the second graphs shows the results for random graphs with 1000 vertices. 
the edge probability. In Figure 2 we see that the graphs that were not solved without the almost bipartite method all have very similar edge probabilities. For random graphs on 1000 vertices, using an edge probability between 0.005 and 0.015 almost guarantees that the resulting random graph cannot be solved without the almost bipartite method. It is more difficult to generate random graphs with 500 vertices that cannot be solved without the almost bipartite method, since only about one out of every thirty graphs with edge probability between 0.03 and 0.045 cannot be solved without using the almost bipartite method.

\subsection{Triangle Elimination}

The triangle elimination is by far the most powerful reduction in our arsenal. It is responsible for eliminating the largest number of both vertices and edges. The triangle elimination works extremely well when the graphs are dense. When the edge probability is more than 0.1 , the triangle elimination usually removes more than 95 percent of both vertices and edges. The result is that, for finding $\frac{3}{2}$-approximate vertex cover, the only interesting random graphs are the graphs that are very sparse. For the dense random graphs, the triangle elimination is simply too effective for those instances to be interesting. Figure 3 shows the fraction of vertices that the triangle elimination removes from the graphs when we use vertex sets of size 500 and 1000. The graphs use a single point for every graph solved so we see how the distribution in the fraction of removed vertices changes with the edge probability and the number of vertices in the graph. By having more vertices, we decrease the variance of the fraction of removed vertices, which indicates that the effect of the reductions gets more consistent as the random graphs get larger.

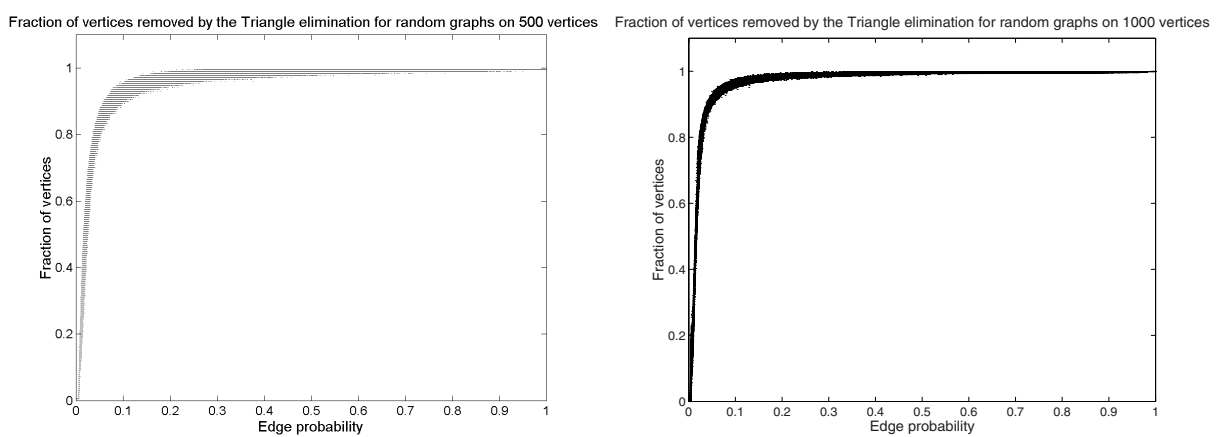

Fig. 3. The fraction of vertices removed by the triangle elimination. The first graph shows the results for randomly generated graphs on 500 vertices and the second graphs shows the results for random graphs with 1000 vertices. The graph show a single point for every solved graph, so the first graph has 1,000,000 points while the second uses 100,000 points. 


\subsection{Extended Network Flow and Low Degree}

The extended network flow method and the low degree eliminations combine to find approximate vertex cover when the graphs are very sparse. When we look at the success of these methods we first need to look at the combined effort since any comparison must take into account which method is used first when we automate the graph reductions to find approximate covers. In our setup, we start by using the extended network flow method, then we use triangle elimination and finally the low degree reductions. This means that if the initial graph is very sparse, the extended network flow method will finish it off easily, while the low degree methods would probably have done the same thing if they were given the chance. Also, by using the low degree methods after we use the triangle elimination, we grant the low degree methods a license to mop up any vertices that remain after the triangle elimination has removed the majority of the graph.
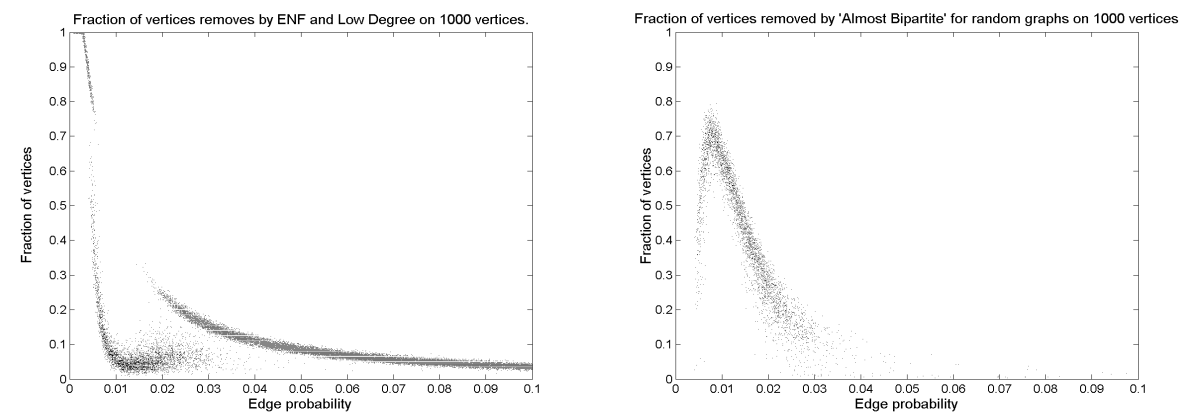

Fig. 4. The fraction of vertices removed from random graphs on 1000 vertices. The first graph shows the combined fraction of vertices removed by the extended network flow method and the low degree methods. The second graph shows the fraction of vertices removed by the almost bipartite method. The graphs are random graphs on 1000 vertices. In both graphs, we focus on edge probabilities less than 0.1 .

The first graph in Figure 4 shows that the performance of the low-degree and extended network flow reductions is consistent for every value of edge probability, except when the edge probability is between 0.005 and 0.02 for random graphs with 1000 vertices. This gap is also obvious in Figure2 since these are the graphs that we could not solve without using the almost bipartite reduction.

\subsection{Almost Bipartite Reduction}

Figure 3 shows that the results for the triangle elimination are consistent, even for the values of edge probability where the extended network flow and the low degree reductions get into trouble. The fraction of vertices drops sharply once the edge probability falls below a certain threshold, but the graph does not show any jumps or erratic behavior. On the other hand, Figure 4 shows how the extended network flow method and the low degree reductions become 
very inconsistent and erratic for certain values of the edge probability, and how the almost bipartite method comes into play when the other reductions are not sufficient to find an approximate vertex cover. We only use the almost bipartite method once the triangle elimination, extended network flow and the low degree reductions have all tried and failed to remove vertices from the graph. When we had to use the almost bipartite method, it worked admirably and found a $\frac{3}{2}$-approximate vertex cover for every single instance of the graphs with 1000 vertices or less.

\subsection{Large Sparse Graphs}

The reductions that we have introduced do not guarantee that we will find an approximate vertex cover for every single graph. However, we have managed to find approximate vertex covers for every single graph we have introduced so far. When we looked at sparse random graphs with 10,000 vertices, we finally found some graphs for which we couldn't find a $\frac{3}{2}$-approximate vertex cover. By using our graph reductions we reduced the size of the graphs but the reductions were not able to finish off the graphs completely. We generated 2000 graphs with 10,000 vertices and edge probability less than 0.1 . We could only solve 1876 of these 2000 graphs using the reductions with approximation ratio less than $3 / 2$. The remaining 124 graphs were solved by adding the five-cycle reduction to the set of reductions, which gives us an approximation ratio of $5 / 3$. We only generated random graphs with edge probability less than 0.1 in order to focus our efforts on the graphs we knew that could be difficult.

The first graph in Figure 5 shows the number of vertices that remain in the graphs when the graph reductions cannot make any more progress. The second graph in Figure 5 shows how many vertices the triangle elimination removed
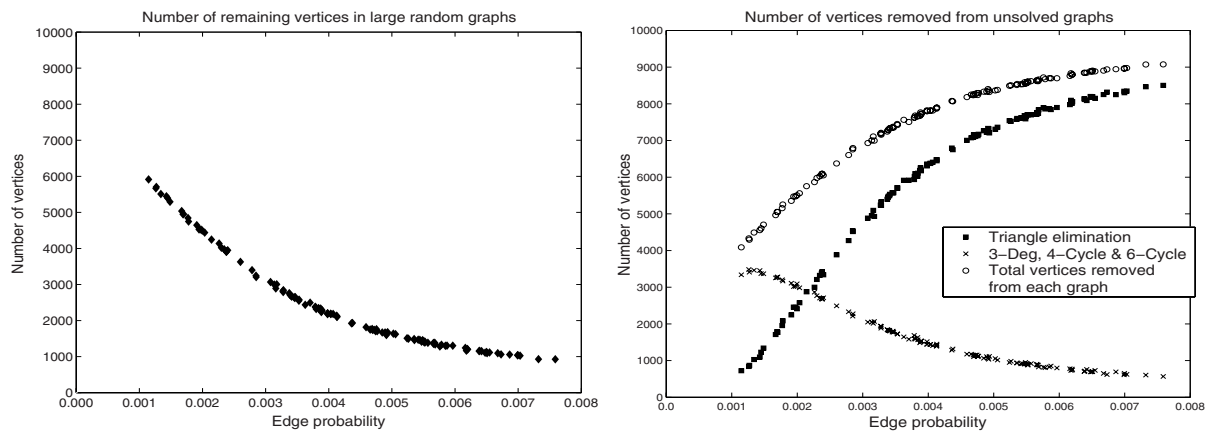

Fig. 5. The graph on the left shows the number of vertices that remain in the graph when our algorithm cannot make any more progress. The graphs are random graphs with 10,000 vertices. The graph on the right shows how many vertices were removed from the graphs in total, how many vertices the triangle elimination removed from the graphs and how many vertices were removed by the 3-degree, 4-cycle and 6-cycle reductions. 
from the graphs and how many vertices were removed by the reductions that create approximated vertices. We used three reductions that create approximated vertices, the 3-degree reduction, 4-cycle reduction and 6-cycle reduction. Figure [5] shows that the number of vertices that remain in the graph mostly depends on how effective the triangle elimination is. The triangle elimination is less effective on sparse graphs, when the graphs become denser the triangle elimination is more effective. We only use the 3-degree reduction, 4-cycles and 6-cycle reductions after the triangle elimination has removed all triangles from the graph. The performance of these 3 reductions is therefore dependent on how many vertices the triangle elimination has removed before we start using the other methods.

\subsection{Comparison with the Optimal Vertex Cover}

We solved some of the random graphs with 200 vertices optimally and compared the size of the optimal vertex cover to the approximate vertex cover that we got from our reductions. The approximation ratio follows a similar pattern as the performance of each reduction, where we find that the edge probabilities of the most interesting graphs are found in a small interval. When the edge probability is too high, the graphs are very dense and the optimal vertex covers are large, which means that any feasible cover is likely to have a good approximation ratio. However, if the edge probability is too small, we can easily solve the graphs optimally using the extended network reduction.

The average approximation ratio over graphs with edge probability $\geq 0.3$ is 1.015 with the largest approximation ratio equal to 1.033. When the graphs are very sparse, where the size of the optimal vertex cover is less than 100, the extended network flow method usually manages to find an optimal cover, so the approximation ratio for these graphs is equal to 1 . There is however an interval in the edge probability where the graph is not too dense and not too sparse, and in that interval there is more variation in the approximation ratio. The highest approximation ratio is 1.074 and we get that when the edge probability is equal to

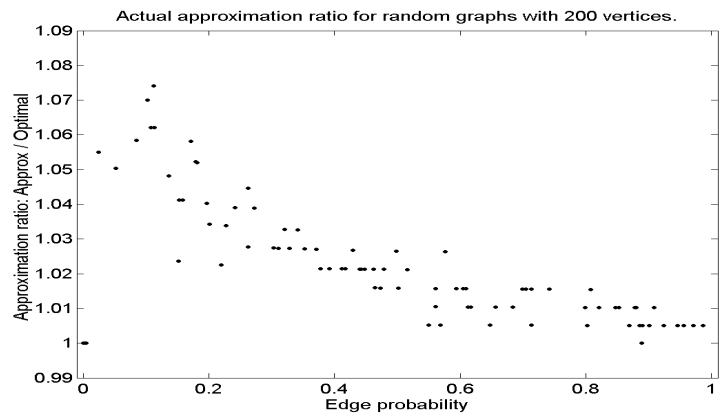

Fig. 6. The ratio of the size of the approximate vertex cover that we get from our reductions over the size of an optimal vertex cover. The graphs are random graphs on 200 vertices. 
0.112. The average approximation ratio for graphs with edge probability between 0.05 and 0.2 is equal to 1.052 . We see that the largest approximation ratio, 1.074, is still very far from our upper limit of 1.5 and the overall average approximation ratio is equal to 1.023 .

\section{Conclusions}

We used a collection of simple reductions where we allowed reductions that have a worst case approximation ratio of $3 / 2$. Even though these reductions do not guarantee that we will find a solution, we ran these reductions on a wide collection of test problems from every source we could find and by combining them we managed to find an approximate vertex cover for almost every single random graph of the that we generated. The reductions that we use are extremely fast and easily applied, and since the bad examples have a very restrictive structure, these reductions should work well in practice.

\section{References}

1. Asgeirsson, E., Stein, C.: Vertex cover approximations: Experiments and observations. WEA, pp. 545-557 (2005)

2. Cook, S.: The complexity of theorem proving procedures. In: Proceedings of the third annual ACM symposium on Theory of computing, pp. 151-158 (1971)

3. Cormen, T.H., Leiserson, C.E., Rivest, R.L., Stein, C.: Introduction to algorithms. MIT Press, Cambridge, MA, USA (2001)

4. Crescenzi, P., Kann, V.: A compendium of NP optimization problems. http://www.nada.kth.se/theory/problemlist.html

5. Dinur, I., Safra, S.: The importance of being biased. In: Proc. 34th Ann. ACM Symp. on Theory of Comp. pp. 33-42 (2002)

6. Erdös, P., Gallai, T.: On the minimal number of vertices representing the edges of a graph. Publ. Math. Inst. Hungar. Acad. Sci. 6, 181-202 (1961)

7. Erdös, P., Rényi, A.: On random graphs. Publ. Math. Debrecen 6, 290-297 (1959)

8. Halperin, E.: Improved approximation algorithms for the vertex cover problem in graphs and hypergraphs. In: Proc. 11th Ann. ACM-SIAM Symp. on Discrete Algorithms, pp. 329-337 (2000)

9. Hochbaum, D.S.: Efficient bounds for the stable set, vertex cover and set packing problems. Discrete Applied Mathematics 6, 243-254 (1983)

10. Karakostas, G.: A better approximation ratio for the vertex cover problem. In: Caires, L., Italiano, G.F., Monteiro, L., Palamidessi, C., Yung, M. (eds.) ICALP 2005. LNCS, vol. 3580, Springer, Heidelberg (2005)

11. Karp, R.M.: Reducibility among combinatorial problems. In: Complexity of Computer Computations, pp. 85-103. Plenum Press (1972)

12. Monien, B., Speckenmeyer, E.: Ramsey Numbers and an Approximation Algorithm for the Vertex Cover Problem. Acta Inf. 22, 115-123 (1985) 